\title{
Predictors of acceptance of offered care management intervention services in a quality improvement trial for dementia
}

\author{
Marwa Kaisey ${ }^{1}$, Brian Mittman ${ }^{2}$, Marjorie Pearson ${ }^{3}$, Karen I. Connor ${ }^{4,5}$, Joshua Chodosh ${ }^{6,7}$, Stefanie D. Vassar ${ }^{4,5}$, \\ France T. Nguyen ${ }^{4}$ and Barbara G. Vickrey ${ }^{3,4,5}$ \\ ${ }^{1}$ School of Medicine, University of Michigan, Ann Arbor, MI, USA \\ ${ }^{2}$ Health Services Research and Development Service (HSR\&D) Sepulveda Center of Excellence, VA Greater Los Angeles Healthcare System, \\ Sepulveda, CA, USA \\ ${ }^{3}$ RAND, Santa Monica, CA, USA \\ ${ }^{4}$ Department of Neurology, UCLA David Geffen School of Medicine, Los Angeles, CA, USA \\ ${ }^{5}$ Department of Neurology, VA Greater Los Angeles Healthcare System, Los Angeles, CA, USA \\ ${ }^{6}$ Geriatric Research Education and Clinical Center (GRECC), VA Greater Los Angeles Healthcare System, Sepulveda, CA, USA \\ ${ }^{7}$ Department of Medicine, Division of Geriatrics, UCLA David Geffen School of Medicine, Los Angeles, CA, USA \\ Correspondence to: Barbara Vickrey, MD, MPH, E-mail: bvickrey@ucla.edu
}

Objective: Care management approaches have been proven to improve outcomes for patients with dementia and their family caregivers (dyads). However, acceptance of services in these programs is incomplete, impacting effectiveness. Acceptance may be related to dyad as well as healthcare system characteristics, but knowledge about factors associated with program acceptance is lacking. This study investigates patient, caregiver, and healthcare system characteristics associated with acceptance of offered care management services.

Methods: This study analyzed data from the intervention arm of a cluster randomized controlled trial of a comprehensive dementia care management intervention. There were 408 patient-caregiver dyads enrolled in the study, of which 238 dyads were randomized to the intervention. Caregiver, patient, and health system factors associated with participation in offered care management services were assessed through bivariate and multivariate regression analyses.

Results: Out of the 238 dyads, 9 were ineligible for this analysis, leaving data of 229 dyads in this sample. Of these, 185 dyads accepted offered care management services, and 44 dyads did not. Multivariate analyses showed that higher likelihood of acceptance of care management services was uniquely associated with cohabitation of caregiver and patient $(p<0.001)$, lesser severity of dementia $(p=0.03)$, and higher patient comorbidity $(p=0.03)$; it also varied across healthcare organization sites.

Conclusions: Understanding factors that influence care management participation could result in increased adoption of successful programs to improve quality of care. Using these factors to revise both program design as well as program promotion may also benefit external validity of future quality improvement research trials. Copyright (C) 2011 John Wiley \& Sons, Ltd.

Key words: dementia; care management; intervention participation

History: Received 4 September 2011; Accepted 9 November 2011; Published online 20 December 2011 in Wiley Online Library (wileyonlinelibrary.com).

DOI: 10.1002 /gps.2830

\section{Introduction}

A growing body of clinical research is yielding an array of evidence-based therapeutic approaches that can produce better outcomes for patients with dementia and their caregivers (Mohide et al., 1990; Teri et al., 2003). Much of the randomized controlled trial evidence, however, has limited external validity, as such 
studies occur under ideal, investigator-controlled conditions, focus solely on a particular therapy, or enroll within highly selective subject samples. Evidence from efficacy-oriented studies such as these is perceived as insufficient to stimulate widespread adoption in routine care settings. Efficacy studies should be followed by effectiveness studies to go beyond the narrow context of efficacy studies, increasing the relevance of the research and likely adoption among broader, more representative populations of patients and caregivers across a healthcare system, region, or country.

Previous research evaluating nonpharmacologic interventions for dementia patients or caregivers has examined selected aspects of participant representativeness and factors influencing the decision to participate in such research (Bass and Noelker, 1987; Verheggen et al., 1998; Murphy et al., 2007; Andren \& Elmstahl, 2008; Dobscha et al., 2009). Less evidence is available regarding factors that influence acceptance of offered services once enrolled. Care management trials have rarely reported on why those who consented to participate in the study declined to partake in offered care management services. Wells et al. (2000) noted that only about $75 \%$ of intervention arm subjects in a depression care management intervention actually received any care management services. A post hoc exploration showed that there was variation in acceptance of care management services among intervention subjects across sites, but reasons for site-specific variations are unknown. More recently, a study (Albers-Heitner et al., 2011) of nurse specialist coordinated care to support general practitioners in the care of patients with urinary incontinence cited other reasons for nonparticipation. Among patients who were randomized to the intervention arm, over one-fourth of patients participated in less than the full series of consultations with the nurse specialist. Reasons given were poor health or too much of a burden.

Although there is a dearth of information about why people with dementia and their caregivers accept offered medical and social services, Katz and colleagues (2000) reported that elderly (over age 70) people with impaired functional status who were married or living with others received more hours of informal care than those who were single. In another study (Schneider et al., 2002), people with dementia who lived with their caregiver were more likely to access healthcare services (e.g., outpatient doctor visits) than those whose caregiver did not reside with them.

In a study of reasons for what the investigators defined as nonparticipation (not completing all five sessions) with a home environmental intervention among a sample of 100 caregivers of people with dementia (Gitlin et al., 1999), reasons provided by caregivers for participating in less than the complete series of sessions included caregiver's illness, death of care recipient, as well as the belief that the caregiver did not need any further help (which was the predominant reason). Being a female caregiver and older caregiver, and having less self-efficacy in handling problem behaviors were each modestly correlated with greater participation, although in multivariable analysis, caregiver self-efficacy was not uniquely associated with participation.

Thus, for an intervention that targets caregivers as well as patients, characteristics of the caregivers and of the relationship between the patient and caregiver, in addition to characteristics of patients and the care setting, may affect whether caregivers and patients elect to accept intervention services. Gitlin and colleagues (1999) proposed control theory (Rothbaum et al., 1982; Schulz et al., 1991) as a model for predicting caregiver participation in a home environmental intervention for dementia. Under control theory, individuals seek to maintain control in challenging situations by changing their environment or their emotional state; thus, greater caregiver depression and lower caregiver self-efficacy would be hypothesized as predictive of greater acceptance of care management.

Several trials of chronic care model-based care management or disease management programs that use nurses or social workers as care managers have been tested and found to significantly improve quality of care for patients with dementia and their caregivers (Belle et al., 2006; Callahan et al., 2006; Vickrey et al., 2006). We sought to explore what factors are associated with acceptance of care management services among those eligible for such programs, as knowledge of these factors is relevant to external validity and dissemination of care management models.

We examined associations between the few previously identified patient, caregiver, or patient-caregiver dyadic relationship factors and acceptance of comprehensive care management services for dementia among a sample from a previous trial, leveraging our ability to explore a host of other factors available from the rich dataset of that trial. We analyzed data from enrolled subjects who were randomized to the intervention arm of this multisite cluster randomized controlled trial of a comprehensive dementia care management program (Vickrey et al., 2006). This intervention nearly doubled adherence to guidelines for dementia care and improved patient health outcomes and caregiver self-efficacy and social support. 
Subjects for the trial were recruited from all patients with dementia identified from administrative records and seen in one of three large healthcare systems in one region of southern California.

\section{Methods}

\section{Study design}

Institutional Review Boards of UCLA and of all three participating healthcare organizations approved study procedures. Eligible patients were identified from administrative records by using the following criteria: (i) age 65 years and older; (ii) diagnosis of dementia using the International Classification of Diseases, 9th revision visit codes; and (iii) having an informal caregiver age 18 years or over. To meet Institutional Review Board requirements for minimizing the risk of contacting individuals who did not have dementia (i.e., for whom the International Classification of Diseases, 9th revision code was an error) and to identify additional potentially eligible subjects, the patients' primary care physician reviewed and then added or removed patients from a list of potentially eligible patients - based on the administrative recordsfor whom that physician was the identified primary care provider; physicians were not required to indicate the reason for excluding a name from the list. We estimated that addition of names occurred less than $5 \%$ of the time; we did not track exclusion of names but estimate it to have been similarly infrequent. To guide selection of the appropriate version of the study's informed consent documents, the physicians were asked to indicate whether each patient had mild dementia or "not mild" dementia.

\section{Analysis sample}

Of 952 eligible patients, 408 patient-caregiver dyads across 18 clinics consented to study participation between August 2001 and November 2002. Although all caregivers were consented, patients were also directly consented if they were identified by the physician as having mild dementia. Study participants were unaware of randomization status until after enrollment. Clinics within each of the three healthcare organizations were paired on the basis of volume and then randomized within each pair to intervention or to usual care arms. Of the 408 dyads enrolled, 238 dyads were in clinics randomized to the care management intervention. After eligible subjects returned an informed consent form and baseline survey by mail, contact information for intervention participants was electronically conveyed by study research assistants to care managers at the appropriate healthcare organization. Specially trained dementia care managers (ranging from one to three per healthcare organization) then contacted the caregiver and patient by telephone to initiate care management services. These services began with a home visit to conduct a structured assessment, which triggered care protocols developed by a healthcare system-wide and community-wide steering committee.

Nine patients had died, moved away, and/or were no longer receiving care from the participating healthcare organizations in the interval between enrollment and initial contact to schedule a home visit by the care manager; these nine dyads were excluded from these analyses, yielding an analysis sample of 229 dyads.

\section{Classification of enrolled intervention arm subjects as accepting care management}

Acceptance of care management was defined as whether or not an intervention dyad received a home visit from a care manager. Reasons for declining an offered initial home visit were abstracted from notes recorded by study staff in enrollment tracking files and from notes recorded by care managers in a computerized care management system (CaseTrakker, IMA Technologies) used by the care managers to plan, conduct, and document care management services and interactions with intervention subjects.

\section{Potential predictors of initiation of care management among enrollees}

Our dataset included variables identified in the few previous studies and additional variables that we examined in an exploratory fashion (Table 1).

Previously identified factors from literature. Factors previously identified in the literature included marital status, age, and gender of caregiver (Gitlin et al., 1999); cohabitation of care recipient and caregiver (Katz et al., 2000); caregiver confidence or self-efficacy (Schneider et al., 2002); and the study site or healthcare organization (Wells et al., 2000). Caregiver confidence was assessed by using a 4 -item caregiver mastery scale (Bass et al., 1996). In our study, all of these variables or measures were assessed by caregiver survey.

Exploratory factors. The baseline survey completed by the caregiver also included patient and caregiver 
Table 1 Comparison of baseline characteristics of patient-caregiver dyads by acceptance of offered care management services $(n=229)$

\begin{tabular}{|c|c|c|c|c|c|}
\hline & \multicolumn{2}{|c|}{$\begin{array}{l}\text { Accepted care } \\
\text { management } \\
\text { services }(n=185)\end{array}$} & \multicolumn{2}{|c|}{$\begin{array}{l}\text { Did not accept } \\
\text { care management } \\
\text { services }(n=44)\end{array}$} & \multirow[b]{2}{*}{$\begin{array}{l}\text { Betweer } \\
\text { group } \\
p \text {-value }\end{array}$} \\
\hline & $N(\%)$ & Mean (SD) & $N(\%)$ & Mean (SD) & \\
\hline \multicolumn{6}{|l|}{ Patient characteristics } \\
\hline Female & $101(54.6)$ & - & $24(54.6)$ & - & 0.99 \\
\hline Age, years & - & $79.6(6.2)$ & - & $81.8(7.3)$ & 0.04 \\
\hline Married or living with someone & $116(62.7)$ & - & $22(50.0)$ & - & 0.12 \\
\hline Nonwhite race/ethnicity & $23(12.6)$ & - & 6 (13.6) & - & 0.85 \\
\hline At least high school graduate & $153(82.7)$ & - & $35(79.3)$ & - & 0.62 \\
\hline Managed care Medicare & 145 (78.4) & - & $38(86.6)$ & - & 0.24 \\
\hline \multicolumn{6}{|l|}{ Dementia status } \\
\hline Duration of diagnosis of dementia, years & - & $2.5(2.9)$ & - & $2.3(2.0)$ & 0.76 \\
\hline Duration of symptoms of dementia, years & - & $4.2(3.6)$ & - & $3.6(2.6)$ & 0.34 \\
\hline Dementia severity score (range $0-17)^{a}$ & - & $5.4(3.2)$ & - & $6.7(4.0)$ & 0.01 \\
\hline \multicolumn{6}{|l|}{ Clinical, behavioral, and quality of life measures } \\
\hline $\begin{array}{l}\text { Charlson Comorbidity Index (range 0-21; higher score } \\
\text { indicates more comorbidity) }\end{array}$ & - & $2.8(1.8)$ & - & $2.2(1.4)$ & 0.08 \\
\hline Health Utilities Index Mark 3 (range 0-1) & - & $0.20(0.29)$ & - & $0.12(0.32)$ & 0.13 \\
\hline $\begin{array}{l}\text { Depressed mood scale score (range 0-100; high score } \\
\text { indicates less depression) }\end{array}$ & - & $79.3(16.0)$ & - & $78.6(18.6)$ & 0.81 \\
\hline $\begin{array}{l}\text { Patient aggression in the prior } 4 \text { weeks (any, as reported } \\
\text { by caregiver) }\end{array}$ & $34(18.4)$ & - & $11(25.0)$ & - & 0.32 \\
\hline Behavior problem documented in medical record & $63(34.1)$ & - & $15(34.1)$ & - & 0.99 \\
\hline $\begin{array}{l}\text { Caregiver input obtained about behavior symptom is } \\
\text { perceived as a problem }(n=78)^{\mathrm{b}}\end{array}$ & $40(63.5)$ & - & $6(40.0)$ & - & 0.10 \\
\hline Cholinesterase inhibitor use & $104(56.2)$ & - & $19(44.2)$ & - & 0.12 \\
\hline \multicolumn{6}{|l|}{ Healthcare utilization (past 6 months) } \\
\hline Monthly healthcare and caregiver costs per patient, \$US & - & $\$ 5,250(8,625)$ & - & $\$ 7,234(11,184)$ & 0.40 \\
\hline Any hospitalization & $33(17.8)$ & - & $9(20.5)$ & - & 0.69 \\
\hline Any stay in nursing home, hospice, or assisted living facility & $21(11.4)$ & - & 13 (29.6) & - & 0.002 \\
\hline Any physician visit ${ }^{\mathrm{C}}$ & $168(90.8)$ & - & $37(84.1)$ & - & 0.19 \\
\hline \multicolumn{6}{|l|}{ Dementia care quality indicators (baseline) } \\
\hline $\begin{array}{l}\text { Mean percent of assessment care processes that } \\
\text { occurred per patient }^{d}\end{array}$ & - & $40.4(20.7)$ & - & $31.2(22.7)$ & 0.01 \\
\hline $\begin{array}{l}\text { Mean percent of treatment care processes that occurred } \\
\text { per patient } \mathrm{e}^{\mathrm{e}}\end{array}$ & - & $31.6(30.9)$ & - & $32.9(30.1)$ & 0.79 \\
\hline $\begin{array}{l}\text { Mean percent of education and support care processes } \\
\text { that occurred per patient }\end{array}$ & - & $52.2(28.9)$ & - & $48.9(29.7)$ & 0.50 \\
\hline $\begin{array}{l}\text { Mean percent of safety care processes that occurred } \\
\text { per patient }{ }^{g}\end{array}$ & - & $21.8(24.8)$ & - & $18.9(27.3)$ & 0.50 \\
\hline \multicolumn{6}{|l|}{ Caregiver characteristics } \\
\hline Female & $125(67.6)$ & - & $29(65.9)$ & - & 0.83 \\
\hline Age, years & - & $65.5(13.1)$ & - & $67.0(14.0)$ & 0.54 \\
\hline Married or living with someone & $154(83.2)$ & - & $37(84.1)$ & - & 0.89 \\
\hline Nonwhite race/ethnicity & $22(11.9)$ & - & $5(11.4)$ & - & 0.92 \\
\hline At least high school graduate & 170 (91.9) & - & $43(97.7)$ & - & 0.17 \\
\hline Caregiver lives with the person with dementia & 141 (76.2) & - & $21(47.7)$ & - & $<0.001$ \\
\hline Relationship to the person with dementia: & - & - & - & - & 0.26 \\
\hline Spouse & $106(57.6)$ & - & $20(45.5)$ & - & - \\
\hline Son/son-in-law or daughter/daughter-in-law & $67(36.4)$ & - & $22(50.0)$ & - & - \\
\hline Other & $12(6.5)$ & - & $2(4.6)$ & - & - \\
\hline Caregiving hours per month & - & $277.4(280.6)$ & - & $180.9(243.9)$ & 0.04 \\
\hline Comorbidity score & - & $3.0(3.6)$ & - & $1.3(2.2)$ & 0.002 \\
\hline \multicolumn{6}{|l|}{ Caregiver burden, social support, mastery, and knowledge } \\
\hline $\begin{array}{l}\text { Caregiving-attributed health strain (range 0-100; higher } \\
\text { score indicates less strain) }\end{array}$ & - & $63.4(23.3)$ & - & $61.3(22.6)$ & 0.59 \\
\hline $\begin{array}{l}\text { Social support (range 0-100; higher score indicates more } \\
\text { social support) }\end{array}$ & - & $65.8(28.5)$ & - & $63.7(30.9)$ & 0.68 \\
\hline $\begin{array}{l}\text { Caregiving mastery score (range 0-100; higher score } \\
\text { indicates more mastery) }\end{array}$ & - & $57.4(26.9)$ & - & $56.0(27.0)$ & 0.69 \\
\hline
\end{tabular}




\begin{tabular}{|c|c|c|c|c|c|}
\hline & \multicolumn{2}{|c|}{$\begin{array}{l}\text { Accepted care } \\
\text { management } \\
\text { services }(n=185)\end{array}$} & \multicolumn{2}{|c|}{$\begin{array}{l}\text { Did not accept } \\
\text { care management } \\
\text { services }(n=44)\end{array}$} & \multirow[b]{2}{*}{$\begin{array}{l}\text { Between } \\
\text { group } \\
p \text {-value }\end{array}$} \\
\hline & $N(\%)$ & Mean (SD) & $N(\%)$ & Mean (SD) & \\
\hline $\begin{array}{l}\text { Caregiver dementia knowledge score (range 0-100; } \\
\text { higher score indicates more knowledge) }\end{array}$ & - & $69.2(14.4)$ & - & $69.5(15.4)$ & 0.89 \\
\hline \multicolumn{6}{|l|}{$\begin{array}{l}\text { Receipt of caregiving assistance or respite/ support } \\
\text { services }\end{array}$} \\
\hline $\begin{array}{l}\text { Received services from a professional home health aide } \\
\text { in the prior } 6 \text { months }\end{array}$ & $9(4.9)$ & - & $5(11.4)$ & - & 0.11 \\
\hline $\begin{array}{l}\text { Received services from a paid professional caregiver in } \\
\text { the prior } 6 \text { months }\end{array}$ & $13(7.0)$ & - & $5(11.4)$ & - & 0.34 \\
\hline $\begin{array}{l}\text { Ever received in-home, volunteer or paid respite } \\
\text { care services }\end{array}$ & $35(18.9)$ & - & $9(20.5)$ & - & 0.82 \\
\hline Ever participated in a caregiver support group & $33(17.8)$ & - & $5(11.4)$ & - & 0.17 \\
\hline $\begin{array}{l}\text { Hours of unpaid unskilled informal caregiving in the prior } \\
6 \text { months }\end{array}$ & - & $1834(1735)$ & - & $1577(1955)$ & 0.39 \\
\hline \multicolumn{6}{|l|}{ Healthcare organization ${ }^{\mathrm{h}}$ : } \\
\hline Site A & $13.0 \%$ & - & $6.8 \%$ & - & 0.11 \\
\hline Site B & $27.0 \%$ & - & $43.2 \%$ & - & - \\
\hline Site C & $60.0 \%$ & - & $52.3 \%$ & - & - \\
\hline
\end{tabular}

${ }^{\text {a}}$ Blessed-Roth Dementia Scale score with higher scores indicating more severe dementia.

${ }^{\mathrm{b}}$ Includes $n=78$ patients for whom a behavior symptom was documented in the medical record.

'Includes geriatricians, family physicians or general internal medicine physicians, neurologists, psychiatrists, and other specialty physicians.

${ }^{\mathrm{d}}$ Assessment guidelines: Assessment of activities of daily living, cognitive status, medical conditions including sleeplessness, behavioral problems or depression, primary caregiver and adequacy of support systems, patient/family culture, and decision-making process.

'Treatment guidelines: Care plan developed; caregiver advised to implement predictable routine of daily activities; caregiver input obtained about whether behavior symptom is perceived as a problem; two or more nonpharmacologic approaches for behavior problems recommended to caregiver; monitoring for outcomes and side effects documented if on behavior medication; caregiver understands behavior or depression medication's benefits, side effects, and indications well.

fEducation and support guidelines: Recommendation or discussion of caregiver support group, dementia-related financial planning and respite care, recommendation or discussion of community agencies, and advance directive discussed or completed and documented.

${ }^{g}$ Safety guidelines: Safe Return Program (for wandering) discussed/recommended; monitoring for abuse recommended; dementia diagnosis reported to county health dept. or nondriving status recommended.

${ }^{\mathrm{h}} \mathrm{Ns}$ are omitted so that specific study sites cannot be identified.

demographic characteristics, duration of diagnosis of dementia, duration of symptoms of dementia, and dementia severity, which was based on the 11-item Blessed-Roth Dementia Scale (Blessed et al., 1968). Patient comorbidity was measured by using the Charlson Comorbidity Index (Charlson et al., 1987), and patient quality of life was measured by using a proxy version of the Health Utilities Index Mark 3 (Torrance et al., 1996).

Patient behavioral problems were measured by a 9-item depressed mood scale (Mungus et al., 1997) and if a caregiver reported any patient aggression problems in the prior 4 weeks (Mungus et al., 1997). Patient healthcare service utilization was reported by caregivers and included hospitalizations, physician visits, and stays in a nursing home and hospice or assisted living facility during the prior 6 months. A monthly healthcare and caregiving cost figure during the prior 6 months was calculated by using units of services reported by caregivers and assigning unit costs (Duru et al., 2009).
Mean adherence to dichotomous dementia quality of care indicators was collected at baseline from the caregiver survey and medical record abstraction. These indicators cover four care dimensions including assessment (six indicators), treatment (six indicators), education and support (three indicators), and safety (three indicators) (Vickrey et al., 2006; Chodosh et al., 2007).

Caregiver comorbidity was calculated as a weighted sum of self-reported chronic conditions from a comorbid health conditions checklist (Hays et al., 1995). Caregiver measures include caregiving-attributed health strain (five items) (Bass et al., 1996), caregiver social support (two items) (Sherbourne \& Stewart, 1991), and knowledge about dementia (five items).

Caregiving assistance and support services were assessed by the caregiver survey, including whether services were received from a professional home health aide or a paid professional caregiver in the prior 6 months and whether in-home respite services 
(paid or volunteer) were ever received. Caregivers also reported if they ever participated in a caregiver support group and the number of unpaid caregiving hours in the prior 6 months for all informal caregivers.

\section{Analysis}

Analyses were conducted by using Stata Version 11 (College Station, TX). Bivariate analyses were conducted by using chi-square tests for categorical variables, two-sample $t$-tests for numerical values, and the Wilcoxon two-sample rank-sum tests for skewed numerical values to compare those dyads who accepted care management services after enrollment with those dyads who did not. To analyze what factors were associated with acceptance of offered participation in the care management program, variables with a bivariate association having a $p$-value $\leq 0.15$ were identified and entered into forward stepwise regression model, allowing variables to enter at $p \leq 0.05$.

Prior to multivariable modeling, predictors with Pearson correlations greater than 0.3 were identified and removed. Regression models were run once with two indicator variables for the three healthcare organization sites (sites A and B as the two indicator variables, and site $\mathrm{C}$ as the reference site), and then a second time with a different site as the reference site (sites $\mathrm{B}$ and $\mathrm{C}$ as the two indicator variables, and site $\mathrm{A}$ as the reference site) to determine differences across all three healthcare organizations. To qualitatively examine why enrolled patients in the intervention arm did not accept care management, we reviewed and categorized comments entered in free text form in study tracking files and in the care management software system.

\section{Results}

Of the 229 intervention dyads in our analysis sample, $185(80.1 \%)$ agreed to and received an initial assessment home visit from the care manager. Bivariate analyses showed that when comparing those study participants who did or did not accept these services, patients in those dyads accepting offered care management were younger $(p=0.04)$; had less severe dementia $(p=0.01)$; were less likely to have used nursing home, hospice, or assisted living services in the prior 6 months $(p=0.002)$; and had higher rates of adherence to dementia indicators on assessment care processes $(p=0.01)$. Caregivers in the group who accepted offered care management services were more likely to live with the person with dementia $(p \leq 0.001)$, had higher comorbidity $(p=0.002)$, and spent more hours caregiving $(p=0.04)$ than those caregivers in the group not accepting offered services, but were otherwise no different across a wide range of measured characteristics (Table 1).

Multivariable modeling showed that cohabitation of caregiver and patient, less severe dementia, greater patient medical comorbidity, and healthcare organization were uniquely associated with higher likelihood of accepting offered care management services (Table 2). Of the 44 intervention dyads who did not accept offered care management services, for 13 dyads, specific reasons were noted in the care management records: two patients had advanced dementia and were perceived by the caregiver as too ill; six caregivers specifically stated they were too busy to participate; and five caregivers stated that they did not feel care management was necessary because the patient was doing well.

\section{Discussion}

We explored potential factors predicting who accepted care management for dementia among those who consented and enrolled in the trial. We found that the strongest predictor was whether the caregiver

Table 2 Factors uniquely associated with acceptance of care management services among intervention arm enrollees $(n=229)$ from multivariable regression model*

\begin{tabular}{llc}
\hline & Odds ratio (95\% Cl) & $p$-value \\
\hline Cohabitation of caregiver and patient $^{a}$ & $3.62(1.77$ to 7.39$)$ & $<0.001$ \\
Less severe dementia $^{a}$ & $1.12(1.03$ to 1.60$)$ & 0.03 \\
Greater patient comorbidity & $1.28(1.03$ to 1.60$)$ & 0.03 \\
Site A vs. Site B & $2.25(0.74$ o 6.83$)$ & 0.04 \\
Site C vs. Site B & $2.76(1.30$ to 5.86$)$ & 0.008 \\
\hline
\end{tabular}

*Stepwise regression included variables from bivariate analysis with a $p$-value $\leq 0.15$ (except for patient marital status that had high Pearson correlations (correlation $=0.52$ ) with caregiver lives with patient); odds ratio $\geq 1$ indicating greater likelihood of trial enrollee participating in the care management intervention services.

${ }^{\mathrm{a} B}$ Blessed-Roth Dementia Scale score: range $0-17$ with higher scores indicating more severe dementia.

${ }^{\mathrm{b}}$ Charlson Comorbidity Index, measured from the medical record abstraction: range 0-21 with higher scores indicating more comorbidity. 
and patient lived together, a factor unique to care management programs that rely heavily on caregivers and involve patients with frailty requiring such dyadic relationships for effective care. There was a modest association of less severe dementia with caregiver acceptance of care management. This finding may reflect that when compared with caregivers of patients with less advanced dementia, caregivers of patients with advanced dementia who had already transitioned to long-term care or to hospice may have perceived a lower need for services to prevent or lessen challenges, because of the already advanced stage of the disease.

Compared with Belle's trial targeting caregiver depression and quality of life (Belle et al., 2006), in which only $2 \%$ of intervention group subjects did not accept offered intervention activities, our study had a lower rate of acceptance of such care management services. However, Belle and colleagues' trial only enrolled caregivers who lived with a relative with dementia, a factor that we found strongly predictive of acceptance of participation in the care management program. Thirty percent of our trial enrollees did not live with a person with dementia. Excluding this population from care management programs would exclude a sizable proportion from quality of care improvement programs.

Also reported in a prior depression care management trial, we found variation across participating sites. This variation could be because of prior experience or length of time the patient was affiliated with his or her healthcare plan or with the primary care physician, or the characteristics of the care manager (Wells et al., 2000).

We did not find an association between acceptance of care management services and either caregivingattributed health strain or caregiver self-efficacy. This lack of association is consistent with one prior study (Gitlin et al., 1999) and adds evidence that control theory does not appear to apply as a model for predicting acceptance of a dementia care program.

Although we identified several factors associated with acceptance of initiation of care management, generalizability of these findings may be limited to dementia, where caregivers play a central role in such interventions and essentially are the decision makers regarding follow-up to receive those care management services.

We note that intervention participation is not the same as recruitment or keeping people engaged in an intervention program once initiated, topics that have received more attention in the literature. Yet, engaging high proportions of study participants to accept invitations into the intervention and actively participate in the quality improvement programs is one of several steps necessary to ensure that an intervention is successfully implemented.

Finally, we note that because the care management model followed a prevention paradigm-intervening to prevent problems rather than to solely react to and manage problems already underway-ways to improve decision making by caregivers that take into account their understanding of future benefit may be useful in improving participation.

\section{Limitations}

Decision making may differ by subjects in a research study and real-world clinical care setting. Because we were conducting a secondary analysis of our existing dataset from this trial, we had not conducted interviews of study participants to purposefully identify themes or factors not available in the care management records dataset. The potential for selection bias exists because primary care physicians reviewed their patient lists generated from administrative data prior to the mailing of study invitation materials and could exclude individuals from being invited to participate in the study. However, we believe this potential bias is small because the specified criteria for excluding names was that the patient did not have a diagnosis of dementia, and only a small proportion of all names generated from the administrative databases for review by these physicians are estimated to have been excluded from the mailing of study invitation materials.

\section{Conclusion}

An important factor influencing the effectiveness of healthcare delivery interventions is individuals' acceptance of offered program services. Existing literature has mainly focused on barriers to recruitment and enrollment into research studies, as opposed to factors explaining the extent of participation in the intervention program. Understanding these factors could result in greater external validity of study findings and increased adoption of successful programs to improve quality of care. We found that patient-caregiver dyads randomized to the intervention arm were more likely to accept care management services if the patient lived with the caregiver, suggesting that greater involvement or perceived need from the caregiver's perspective positively influenced accepting the offered services. 


\section{Key points}

- Over three-fourths of enrollees in a trial who were randomized to receive a dementia care management program engaged in offered services.

- Higher likelihood of acceptance of care management services was uniquely associated with cohabitation of caregiver and patient (strongest association), less severe dementia, and higher patient medical comorbidity; likelihood of acceptance also varied by site.

- For future initiatives, cohabitation of patient and caregiver may be an important stratification criterion for the type of program offered.

- Future programs may benefit from specifically alerting caregivers and care recipients to anticipated future need even when current need is perceived as low.

\section{Acknowledgements}

We would like to remember Martin Lee, $\mathrm{PhD}$, who provided suggestions about the statistical approach. The authors thank the many partners in the ACCESS study and in particular, Theodore Ganiats, MD of UCSD, Lisa Heikoff, MD of Kaiser Permanente, Richard Della Penna, MD of Kaiser Permanente, and Robert DeMonte, MD of Scripps.

This analysis was supported by the California Department of Public Health (contract 06-55314) for the Alzheimer's Disease Research Center of California.

\section{Conflicts of interest}

Drs. Mittman, Connor, Pearson, and NguyenGrozavu, and Ms. Kaisey and Vassar have no conflicts of interest to report. Dr. Chodosh received a grant from the SCAN Foundation. Dr. Vickrey serves as a consultant for EMD Serono Canada, Inc.

\section{References}

Albers-Heitner PC, Lagro-Janssen TA, Joore MM, et al. 2011. Effectiveness of involving a nurse specialist for patients with urinary incontinence in primary care: results of a pragmatic multicentre randomized controlled trial. Intl J Clin Pract 65: 635-637.
Andren S, Elmstahl S. 2008. Psychosocial intervention for family caregivers of people with dementia reduces caregiver's burden: development and effect after 6 and 12 months. Scand J Caring Sci 22: 98-109.

Bass DM, Noelker LS. 1987. The influence of family caregivers on elder's use of in-home services: an expanded conceptual framework. J Health Soc Behav 28: 184-196.

Bass DM, Noelker LS, Rechlin LR. 1996. The moderating influence of service use on negative caregiving consequences. J Gerontol B Psychol Sci Soc Sci 51: S121-S131.

Belle SH, Burgio L, Burns R, et al. 2006. Enhancing the quality of life of dementia care givers from different ethnic or racial groups: a randomized controlled trial. Ann Intern Med 145: 727-738.

Blessed G, Tomlinson BE, Roth M. 1968. The association between quantitative measure of dementia and of senile state in the cerebral grey matter of elderly subjects. Br J Psychiatry 114: 797-811.

Callahan CM, Boustani MA, Unverzagt FW, et al. 2006. Effectiveness of collaborative care for older adults with Alzheimer disease in primary care: a randomized controlled trial. JAMA 295: 2148-2157.

Charlson ME, Pompei P, Ales KL, MacKenzie CR. 1987. A new method of classifying prognostic comorbidity in longitudinal studies: development and validation. $J$ Chronic Dis 40: 373-383.

Chodosh J, Mittman BS, Connor KI, et al. 2007. Caring for patients with dementia: how good is the quality of care? Results from three health systems. JAGS 55: 1260-1268.

Dobscha SK, Corson K, Perrin NA, et al. 2009. Collaborative care for chronic pain in primary care: a cluster randomized trial. JAMA 301: 1242-1252.

Duru OK, Ettner SL, Vassar SD, Chodosh J, Vickrey BG. 2009. Cost evaluation of a coordinated care management intervention for dementia. Am J Manag Care 15: $521-528$.

Gitlin LN, Cocoran M, Winter L, Boyce A, Marcus S. 1999. Predicting participation and adherence to a home environmental intervention among family caregivers of persons with dementia. Family Relations 48: 363-372.

Hays RD, Well KB, Sherbourne CD, Rogers W, Spritzer K. 1995. Functioning and well-being outcomes of patients with depression compared with chronic general medical illnesses. Arch Gen Psychiatry 52: 11-19.

Katz SJ, Kabeto M, Langa KM. 2000. Gender disparities in the receipt of home care for elderly people with disability in the United States. JAMA 284: 3022-3027.

Mohide EA, Pringle DM, Streiner DL, et al. 1990. A randomized trial of family caregiver support in the home management of dementia. J Am Geriatr Soc 38: 446-454.

Mungus D, Geck LC, Hargrave R, et al. 1997. Homogeneous Patterns of Behavioral Feature of Dementia: Caregiver and Clinical Ratings. Department of Neurology, University of California, Davis: Davis, CA.

Murphy MR, Escamilla MI, Blackwell PH, et al. 2007. Assessment of caregivers' willingness to participate in an intervention research study. Res Nurs Health 30: $347-355$.

Rothbaum F, Weisz TR, Snyder S. 1982. Changing the world and changing the self: a two-process model of perceived control. J Personality Social Psych 42: 5-37.

Schneider J, Hallam A, Murray J, et al. 2002. Formal and informal care for people with dementia: factors associated with service receipt. Aging Ment Heath 6: $255-265$.

Schulz R, Heckhansen J, Locher JL. 1991. Adult development, control, and adaptive functioning. J Social Iss 47: 177-196.

Sherbourne CD, Stewart AL. 1991. The MOS social support survey. Soc Sci Med 32: $705-714$.

Teri L, Gibbons LE, MCurrey SM, et al. 2003. Exercise plus behavioral management in patients with Alzheimer disease: a randomized controlled trial. JAMA 290: 2015-2022.

Torrance GW, Feeny DH, Furlong WJ, et al. 1996. Multiattribute utility function for a comprehensive health status classification system. Health Utilities Index Mark 2. Med Care 34: 702-722.

Verheggen FW, Nieman F, Jonkers R. 1998. Determinants of patient participation in clinical studies requiring informed consent: why patients enter a clinical trial. $\mathrm{Pa}$ tient Educ Couns 35: 111-125.

Vickrey BG, Mittman BS, Connor KI, et al. 2006. The effect of a disease management intervention on quality and outcomes of dementia care: a randomized, controlled trial. Ann Intern Med 145: 713-726.

Wells KB, Sherbourne C, Schoenbaum M, et al. 2000. Impact of disseminating quality improvement programs for depression in managed primary care: a randomized controlled trial. JAMA 283: 212-220. 